\title{
ON THE ESTIMATION OF ROBUST STABILITY REGIONS FOR NONLINEAR SYSTEMS WITH SATURATION
}

\author{
Daniel F. Coutinho* \\ daniel@ee.pucrs.br
}

\author{
Daniel J. Pagano ${ }^{\dagger}$ \\ danieldas.ufsc.br
}

\author{
Alexandre Trofino ${ }^{\dagger}$ \\ trofinoddas.ufsc.br \\ *Department of Electrical Engineering, Pontifícia Universidade Católica do Rio Grande do Sul, \\ Av. Ipiranga 6681, 90619900, Porto Alegre, RS, Brazil. \\ ${ }^{\dagger}$ Department of Automation and Systems, Universidade Federal de Santa Catarina, \\ PO Box 476, 88040900, Florianópolis, SC, Brazil.
}

\begin{abstract}
This paper addresses the problem of determining robust stability regions for a class of nonlinear systems with timeinvariant uncertainties subject to actuator saturation. The unforced nonlinear system is represented by differentialalgebraic equations where the system matrices are allowed to be rational functions of the state and uncertain parameters, and the saturation nonlinearity is modelled by a sector bound condition. For this class of systems, local stability conditions in terms of linear matrix inequalities are derived based on polynomial Lyapunov functions in which the Lyapunov matrix is a quadratic function of the state and uncertain parameters. To estimate a robust stability region is considered the largest level surface of the Lyapunov function belonging to a given polytopic region of state. A numerical example is used to demonstrate the approach.
\end{abstract}

KEYWORDS: Nonlinear systems, stability region, uncertainty, convex optimization, saturation.

\section{RESUMO}

Este artigo trata do problema de determinar regiões de esta-

Artigo submetido em 12/12/02

1a. Revisão em 03/07/03

Aceito sob recomendação do Ed. Assoc. Prof. Liu Hsu bilidade robustas para uma classe de sistemas não lineares com incertezas invariantes no tempo e sujeitos à saturação no sinal de controle. O sistema não linear é representado por uma equação algébrico- diferencial onde as matrizes do sistema são funções racionais dos estados e incertezas e a saturação no controle é representada como uma condição de setor. As condições de estabilidade local propostas são expressas por LMIs e estão baseadas numa função de Lyapunov que é polinomial (ordem 4) nos estados e quadrática nos parâmetros incertos. Para estimar a região de estabilidade robusta propõe-se um problema de maximização da maior curva de nível da função de Lyapunov dentro de um politopo dado representando as condições iniciais. Os resultados são ilustrados através de um exemplo numérico.

PALAVRAS-CHAVE: Sistemas não-lineares, região de estabilidade, incerteza, otimização convexa, saturação.

\section{INTRODUCTION}

Actuator saturation appears frequently in feedback control systems and its presence can lead the system to parasitic equilibrium points, limit cycles and other more complex phenomena. In the control literature, many researchers have addressed the problem of estimating stability regions for open-loop unstable linear systems with bounded inputs by means of the Lyapunov theory and the linear matrix inequal- 
ity (LMI) framework. For instance, Hindi and Boyd (1998) uses the circle and Popov criteria, Gomes da Silva Jr. and Tarbouriech (1999) considers polyhedral Lyapunov functions and Johansson (2002) employs piecewise techniques. However, there are few results for the nonlinear case such as the works of Barreiro et al. (2002) which combines the bifurcation analysis and Lyapunov theory and Bean et al. (2002) which uses piecewise bilinear models and a single polynomial Lyapunov function.

On the other hand, the stability and performance analysis, and control synthesis of uncertain nonlinear systems has been recently addressed by many authors via convex optimization problems, e.g. the works of (El Ghaoui and Scorletti, 1996; Dussy and El Ghaoui, 1997; Chesi et al., 2002) and (Trofino, 2000; Johansen, 2000; Coutinho, Trofino and $\mathrm{Fu}, 2002)$ that consider quadratic and polynomial Lyapunov functions, respectively. In general, non-quadratic Lyapunov functions are less conservative for dealing with uncertain nonlinear systems than the quadratic ones at the expense of extra computations Johansen (2000). Also, the LMI framework has some advantages over other approaches since it can handle parameter-dependent Lyapunov functions, uncertainties, equality and inequality constraints and so on in a numerical tractable way Boyd et al. (1994).

In this scenario, the purpose of this paper is to derive robust conditions in terms of LMIs for analyzing the stability of (open-loop unstable) nonlinear systems with time-invariant uncertainties and subject to input saturation based on the work of Trofino (2000) which have proposed a convex approach to the domain of attraction problem for rational nonlinear systems. To this end, the unforced system is described by rational differential-algebraic equations and the saturation nonlinearity is modelled by a sector bound condition. Using a polynomial Lyapunov function, we give sufficient local stability conditions for the saturated systems while providing an estimate of its stability region (SR) for all possible admissible uncertainty. An uncertain controlled pendulum system with input saturation is used to show the potential of our approach.

The rest of this paper is as follows. Section 2 states the problem of interest and Section 3 introduces some preliminary results. In the sequel, Section 4 presents the main result of this paper, Section 5 gives a numerical example and Section 6 ends the paper.

The notation used throughout this paper is standard. $\mathbb{R}^{n}$ denotes the set of $n$-dimensional real vectors, $\mathbb{R}^{n \times m}$ is the set of $n \times m$ real matrices, $I_{n}$ is the $n \times n$ identity matrix, $0_{n \times m}$ is the $n \times m$ matrix of zeros and $0_{n}$ is the $n \times n$ matrix of zeros. For a real matrix $S, S^{\prime}$ denotes its transpose, and $S>0$ means that $S$ is symmetric and positive-definite. The time derivative of a function $r(t)$ will be denoted by $\dot{r}(t)$ and the argument $(t)$ is often omitted. The symbol $\star$ for a block matrix represents its symmetrical block outside the main diagonal. For two polytopes $\mathcal{B}_{1} \subset \mathbb{R}^{n_{1}}$ and $\mathcal{B}_{2} \subset \mathbb{R}^{n_{2}}$ the notation $\mathcal{B}_{1} \times \mathcal{B}_{2}$ represents that $\left(\mathcal{B}_{1} \times \mathcal{B}_{2}\right) \subset \mathbb{R}^{\left(n_{1}+n_{2}\right)}$ is a metapolytope obtained by the cartesian product, and $\mathcal{V}\left(\mathcal{B}_{1} \times \mathcal{B}_{2}\right)$ represents the set of all vertices of $\mathcal{B}_{1} \times \mathcal{B}_{2}$. Matrix and vector dimensions are omitted whenever they can be inferred from the context.

\section{PROBLEM STATEMENT}

Consider the following nonlinear system

$$
\left\{\begin{aligned}
\dot{x} & =f(x, \tau, \lambda)+g(x, \tau, \lambda, u) \\
0 & =h(x, \tau, \lambda) \\
u & =\operatorname{sat}\left(K^{\prime} x\right)
\end{aligned}\right.
$$

where $x \in \mathcal{B}_{x} \subset \mathbb{R}^{n}$ denotes the state, $\tau \in \mathcal{B}_{\tau} \subset \mathbb{R}^{l}$ denotes the vector of algebraic variables, $\lambda \in \mathbb{R}^{p}$ denotes the vector of constant uncertain parameters associated to disturbances, $u \in \mathbb{R}$ is the control input, sat(.) is the unit saturation function, $K \in \mathbb{R}^{n}$ is a given constant vector such that system (1) is locally stable, $\mathcal{B}_{x}$ is a known polytopic region of state containing the origin, and $\mathcal{B}_{\tau}$ represents the set of admissible algebraic variables. We assume for the above system that:

A1 The uncertain parameters represented by $\lambda$ lie in a given polytope $\mathcal{B}_{\lambda}$, i.e. $\lambda \in \mathcal{B}_{\lambda}$.

A2 The nonlinear vectors $f(x, \tau, \lambda), g(x, \tau, \lambda, u)$ and $h(x, \tau, \lambda)$ are continuous on their arguments and bounded for all $(x, \tau, \lambda) \in \mathcal{B}_{x} \times \mathcal{B}_{\tau} \times \mathcal{B}_{\lambda}$.

A3 The origin is an equilibrium point for all admissible uncertainty, i.e. $f(0, \tau, \lambda)=0$.

A4 The unit saturation function is described by

$$
\operatorname{sat}\left(K^{\prime} x\right)=\left\{\begin{array}{ccc}
1 & & K^{\prime} x>1 \\
K^{\prime} x & \text { if } & \left|K^{\prime} x\right| \leq 1 \\
-1 & & K^{\prime} x<-1
\end{array}\right.
$$

Considering the above assumptions, the purpose of this paper is to analyze the local stability of the origin of system (1) while providing an estimate of its SR (stability region) in a numerical tractable manner. To this end, we will use polynomial Lyapunov functions which will be obtained by means of a convex optimization problem in terms of LMIs.

We end this section presenting the following basic result from the Lyapunov theory Kiyama and Iwasaki (2000, Lemma 1). 
Lemma 1 Consider a nonlinear system $\dot{x}=f(x, \tau, \lambda)$ where $f: \mathcal{B}_{x} \times \mathcal{B}_{\tau} \times \mathcal{B}_{\lambda} \mapsto \mathbb{R}^{n}$ is a continuous function such that $f(0, \tau, \lambda)=0$. Suppose there exist positive scalars $\epsilon_{1}, \epsilon_{2}, \epsilon_{3}$ and a continuously differentiable function $V: \mathcal{B}_{x} \times \mathcal{B}_{\lambda} \mapsto \mathbb{R}$ satisfying the following conditions:

$$
\begin{gathered}
\epsilon_{1} x^{\prime} x \leq V(x, \lambda) \leq \epsilon_{2} x^{\prime} x, \forall(x, \lambda) \in \mathcal{B}_{x} \times \mathcal{B}_{\lambda} \\
\dot{V}(x, \lambda) \leq-\epsilon_{3} x^{\prime} x, \forall(x, \tau, \lambda) \in \mathcal{B}_{x} \times \mathcal{B}_{\tau} \times \mathcal{B}_{\lambda} \\
\mathcal{R} \triangleq\{x: V(x, \lambda) \leq 1\} \subset \mathcal{B}_{x}, \forall \lambda \in \mathcal{B}_{\lambda}
\end{gathered}
$$

Then, $V(x, \lambda)$ is a Lyapunov function in $\mathcal{B}_{x} \times \mathcal{B}_{\lambda}$. Moreover, for all $x(0) \in \mathcal{R}$ the trajectory $x(t)$ belongs to $\mathcal{R}$ and approaches the origin as $t \rightarrow \infty$.

\section{PRELIMINARIES}

Before stating the main result of this paper, we introduce in the following some preliminary results in order to obtain a convex characterization of Lemma 1.

\subsection{Sector Bound Condition}

One way to deal with the saturation nonlinearity is to restrict the amplitude of the input signal leading to a constraint on the system state Hindi and Boyd (1998). Letting $\rho \geq 0$ be the allowable input amplitude over the saturation level, hereafter called the level of over saturation, the constraint

$$
|u(t)| \leq 1+\rho
$$

holds if and only if the system state belongs to the set

$$
\mathcal{X}_{\rho} \triangleq\left\{x:\left|K^{\prime} x\right| \leq 1+\rho\right\}
$$

Note when $\rho=0$ (i.e. $x \in \mathcal{X}_{0}$ ) that system (1) behaves with the following dynamics:

$$
\dot{x}=f(x, \tau, \lambda)+g\left(x, \tau, \lambda, K^{\prime} x\right), 0=h(x, \tau, \lambda),
$$

and then we can apply the technique proposed in Coutinho, Bazanella, Trofino and Silva (2002) with the additional constraint $\mathcal{R} \subset \mathcal{X}_{0}$ for analyzing its regional stability. However, the state vector frequently converges to the origin from an initial point outside the set $\mathcal{R} \subset \mathcal{X}_{0}$ and thus the above analysis may be too conservative.

A more appropriate approach is to allow a certain level of saturation with $\rho>0$ using the circle criterion. As a result, we have the following sector bound condition Kiyama and Iwasaki (2000):

$$
\left(u-K^{\prime} x\right)\left(u-\frac{1}{1+\rho} K^{\prime} x\right) \leq 0, \forall x \in \mathcal{X}_{\rho}
$$

Using the well-known $\mathcal{S}$-procedure (Yakubovich, 1971; Boyd et al., 1994), we can add the sector condition (7) into the Lyapunov inequality (4). Thus, there exists a positive scalar $\mu$ such that the following inequality is satisfied for all $(x, \tau, \lambda) \in \mathcal{B}_{x} \times \mathcal{B}_{\tau} \times \mathcal{B}_{\lambda}$ and $x \in \mathcal{X}_{\rho}$ :

$$
\dot{V}(x, \lambda)-\mu u^{2}+2 \mu \rho_{1} u^{\prime} K^{\prime} x-\mu \rho_{2} x^{\prime} K K^{\prime} x \leq-\epsilon_{3} x^{\prime} x
$$

where

$$
\rho_{1}=\frac{(2+\rho)}{2(1+\rho)} \text { and } \rho_{2}=\frac{1}{(1+\rho)}
$$

Remark 1 The sector condition in (7) is satisfied for all $x \in \mathcal{X}_{\rho}$, and thus the modified Lyapunov inequality in (8) must be tested in the meta-set $\left(\mathcal{B}_{x} \cap \mathcal{X}_{\rho}\right) \times \mathcal{B}_{\tau} \times \mathcal{B}_{\lambda}$. As a consequence, there exists a compromise between $\mathcal{B}_{x}$ and $\mathcal{X}_{\rho}$ since they define the state domain in which the stability conditions will be checked. In other words, we have to choose the parameter $\rho$ such that the size of $\mathcal{B}_{x} \cap \mathcal{X}_{\rho}$ is maximized. This point will be addressed later on this Section and also in Section 5 by means of an illustrative example.

\subsection{System Model Representation}

Consider that the unforced system in (1) can be rewritten as indicated bellow:

$$
\left\{\begin{aligned}
\dot{x} & =A_{1}(x, \tau, \lambda) x+A_{2}(x, \tau, \lambda) \xi \\
& +B_{1}(x, \tau, \lambda) u+B_{2}(x, \tau, \lambda) \phi \\
0 & =\Omega_{1}(x, \tau, \lambda) x+\Omega_{2}(x, \tau, \delta) \xi \\
0 & =\Phi_{1}(x, \tau, \lambda) u+\Phi_{2}(x, \tau, \lambda) \phi
\end{aligned}\right.
$$

where the vectors $\xi \in \mathbb{R}^{m}$ and $\phi \in \mathbb{R}^{q}$ are nonlinear functions of $(x, \tau, \lambda)$, and the matrices $A_{1}(\cdot), A_{2}(\cdot), B_{1}(\cdot), B_{2}(\cdot)$, and $\Omega_{1}(\cdot) \in \mathbb{R}^{r \times n}, \Omega_{2}(\cdot) \in \mathbb{R}^{r \times m}, \Phi_{1}(\cdot) \in \mathbb{R}^{z}, \Phi_{2}(\cdot) \in$ $\mathbb{R}^{z \times q}$ are affine functions of $(x, \tau, \lambda)$. Throughout this work, we may use $A_{1}(\cdot), A_{2}(\cdot), B_{1}(\cdot), B_{2}(\cdot), \Omega_{1}(\cdot), \Omega_{2}(\cdot), \Phi_{1}(\cdot)$ and $\Phi_{2}(\cdot)$ without their respective dependence on $x, \tau, \lambda$ and $t$ (time) in order to simplify the notation.

In order to guarantee that system (10) is well-posed, we further assume:

A5 The matrices $\Omega_{2}, \Phi_{2}$ in (10) have full column rank for all $x, \tau$ and $\lambda$ of interest.

The above assumption ${ }^{1}$ implies that $\xi$ and $\phi$ can be eliminated from (10) to recover the original system representation in (1), i.e., one can return to the original system representation by defining $\xi$ and $\phi$ in (10) as follows

$$
\xi=-\left(\Omega_{2}^{\prime} \Omega_{2}\right)^{-1} \Omega_{2}^{\prime} \Omega_{1} x, \phi=-\left(\Phi_{2}^{\prime} \Phi_{2}\right)^{-1} \Phi_{2}^{\prime} \Phi_{1} u .
$$

\footnotetext{
${ }^{1} \mathbf{A 5}$ is an usual assumption for descriptor systems, see e.g. Bender and Laub (1997).
} 
Observe that the nonlinear decomposition (10) has an augmented space $\left(\mathbb{R}^{n} \subseteq \mathbb{R}^{n+m}\right)$ and the relationships between $(\xi, \phi)$ and $(x, \tau, \lambda, u)$ are defined by means of the constraints $\Omega_{1} x+\Omega_{2} \xi=0$ and $\Phi_{1} u+\Phi_{2} \phi=0$. As a result, the system can only have rational nonlinearities without singularities at origin in the differential-algebraic equations (El Ghaoui and Scorletti, 1996). However, we can transform a certain differential-algebraic representation with non-rational terms into an augmented differential-algebraic form without nonrational nonlinearities. To illustrate this procedure, consider the following example.

Example 1 Consider a controlled pendulum system whose dynamics is given by

$$
\dot{x}_{1}=x_{2}, \dot{x}_{2}=\lambda \sin x_{1}-x_{2}+u
$$

where $u$ is the control input and $\lambda \in \mathcal{B}_{\lambda}$ is a constant uncertain parameter.

Assume for above system that the angle $x_{1}$ is bounded by $-\pi<x_{1} \leq \pi$. In order to rewrite the above system in the form (10), we define the following auxiliary variables

$$
x_{3}=\sin x_{1} \text { and } \tau=\cos x_{1}
$$

With these auxiliary variables, one can construct: a differential equation $\dot{x}_{3}=\tau x_{2}$ and an algebraic one $x_{3}^{2}+\tau^{2}=1$. Leading to the following augmented system.

$$
\left\{\begin{aligned}
\dot{x}_{1} & =x_{2} \\
\dot{x}_{2} & =-x_{2}+\lambda x_{3}+u \\
\dot{x}_{3} & =\tau x_{2} \\
0 & =x_{3}^{2}+\tau^{2}-1
\end{aligned}\right.
$$

Notice in (12) that we choose $x_{3}=\sin x_{1}$ aiming a rational (augmented) representation of system (11). As a result, a new differential equation have been added to the system whose dynamics depends on the algebraic variable $\tau=\cos x_{1}$ leading to a rational differential-algebraic representation as in (10) of the original system (11).

Finally, rewriting (13) as (10) give rise the following system representation

$$
\left\{\begin{aligned}
\dot{x} & =A_{1} x+A_{2} \xi+B_{1} u \\
0 & =\Omega_{1} x+\Omega_{2} \xi
\end{aligned}\right.
$$

where $x=\left[\begin{array}{lll}x_{1} & x_{2} & x_{3}\end{array}\right]^{\prime}, \xi=\left[\begin{array}{ccc}\tau x_{2} & \tau & 1\end{array}\right]^{\prime}$ and

$$
\begin{gathered}
B_{1}=\left[\begin{array}{l}
0 \\
1 \\
0
\end{array}\right], A_{1}=\left[\begin{array}{ccc}
0 & 1 & 0 \\
0 & -1 & \lambda \\
0 & 0 & 0
\end{array}\right], A_{2}=\left[\begin{array}{lll}
0 & 0 & 0 \\
0 & 0 & 0 \\
1 & 0 & 0
\end{array}\right], \\
\Omega_{1}=\left[\begin{array}{ccc}
0 & \tau & 0 \\
0 & 0 & 0 \\
0 & 0 & x_{3} \\
& I_{3} & \\
& \tau I_{3} & \\
0 & 0 & 0
\end{array}\right], \Omega_{2}=\left[\begin{array}{ccc}
-1 & 0 & 0 \\
1 & -x_{2} & 0 \\
0 & \tau & -1 \\
0 & 0 & -x \\
0 & -x & 0 \\
0 & 1 & -\tau
\end{array}\right] .
\end{gathered}
$$

Observe that $\Omega_{1}$ and $\Omega_{2}$ as given above define the following constraints over $x$ and $\xi$ :

$$
\begin{gathered}
\tau x_{2}-\xi_{1}=0, \xi_{1}-x_{2} \xi_{2}=0, x_{3}^{2}+\tau \xi_{2}-1=0, \\
x-x \xi_{3}=0, \tau x-x \xi_{2}=0, \xi_{2}-\tau \xi_{3}=0,
\end{gathered}
$$

where $\xi_{i}$ are the i-th elements of $\xi$.

It should be noted that the trajectories of system (14) include all trajectories of the original one defined in (11). In particular, suppose that $x_{1}(0), x_{2}(0)$ are the initial conditions of system (11). Then, for the initial conditions $x_{1}(0), x_{2}(0)$ and $x_{3}(0)=\sin x_{1}(0)$, both systems have equal trajectories in the $x_{1}, x_{2}$ sub-space.

Remark 2 The choice of matrices $A_{1}, \ldots, B_{2}$ in (10) is not unique and until now there is no a systematic way to define them. As a result, a bad choice of them can lead to a poor stability region estimate or even fail to provide the system stability (Huang and Lu, 1996). A possible way of reducing this potential conservativeness is to add free multipliers to the problem reducing the dependence on the choice of the system matrices as proposed by Huang and Jadbabaie (1999) and Trofino (2000) using different approaches. In this paper, we follow the technique of Trofino (2000) to handle statedependent LMIs as proposed in Section 4.

\subsection{Lyapunov Function Candidate}

Consider the following Lyapunov function candidate

$$
\begin{gathered}
V(x, \lambda)=x^{\prime} \mathcal{P}(x, \lambda) x, \\
\mathcal{P}(x, \lambda)=\left[\begin{array}{c}
\Theta(x, \lambda) \\
I_{n}
\end{array}\right]^{\prime} P\left[\begin{array}{c}
\Theta(x, \lambda) \\
I_{n}
\end{array}\right],
\end{gathered}
$$

where $P$ is a symmetric matrix to be determined and $\Theta(x, \lambda) \in \mathbb{R}^{v \times n}$ is a given affine matrix function of $(x, \lambda)$.

From the above definition, we can represent $\Theta(x, \lambda)$ as follows:

$$
\Theta(x, \lambda)=\sum_{j=1}^{n} T_{j} x_{j}+\sum_{j=1}^{p} U_{j} \lambda_{j}+Y
$$

where $T_{j}, U_{j}, Y$ are constant matrices with the same dimensions of $\Theta(x, \lambda)$, and $x_{j}, \lambda_{j}$ stand for the elements of the vectors $x$ and $\lambda$, respectively.

To determine the time-derivative of $V(x, \lambda)$, we need to compute the following term:

$$
\frac{d(\Theta(x, \lambda) x)}{d t}=\dot{\Theta}(x, \lambda) x+\Theta(x, \lambda) \dot{x}
$$

Straightforwardly from (16), the term $\dot{\Theta}(x, \lambda) x$ is given by:

$$
\dot{\Theta}(x, \lambda) x=\sum_{j=1}^{n} T_{j} \dot{x}_{j} x=\sum_{j=1}^{n} T_{j} x s_{j} \dot{x}=\tilde{\Theta}(x) \dot{x}
$$


where the matrix $\tilde{\Theta}(x)$ is as follows:

$$
\tilde{\Theta}(x)=\sum_{j=1}^{n} T_{j} x s_{j}
$$

with $s_{j}$ denoting the $j$-th row of the identity matrix $I_{n}$.

Then, using (16), (17) and (18) we can obtain a convex characterization of (8) in terms of LMIs similarly to the procedure proposed in Trofino (2000). We make this point clear later in the proof of Theorem 2.

Remark 3 In spite of the fact that $V(x, \lambda)$ as defined in (15) has a 4th degree in $x$, we have named it as a polynomial Lyapunov function. Notice that the proposed approach can be in a similar way extended for higher polynomial degrees at the cost of more intensive computations, see e.g. (Coutinho and Trofino, 2002). Based on our recent results such as (Coutinho, Trofino and Fu, 2002) and (Coutinho, Bazanella, Trofino and Silva, 2002), the class of Lyapunov function defined in (15) is the one that achieves the best results regarding conservativeness and computational effort.

\subsection{Stability Region}

One of the advantages of using polynomial Lyapunov functions is that they may provide a non-ellipsoidal and thus less conservative estimate of stability regions (SRs). Based on the results of Trofino (2000), we will present in the following the main ideas for estimating robust stability regions.

Firstly, represent the polytope $\mathcal{B}_{x}$ by a set of scalar inequalities as follows:

$$
\mathcal{B}_{x}=\left\{a_{k}^{\prime} x \leq 1, k=1, \ldots, n_{e}\right\}
$$

where $n_{e}$ is the number of edges of $\mathcal{B}_{x}$. It turns out that $\mathcal{B}_{x}$ can also be represented by its vertices.

Now, consider the following set as an estimate of stability region:

$$
\mathcal{R}=\left\{x: x^{\prime} \mathcal{P}(x, \lambda) x \leq 1\right\}
$$

whose boundary is a level surface of the Lyapunov function candidate.

Observe that conditions (3) and (8) (with $\mathcal{R} \subset \mathcal{X}_{\rho}$ ) implies that $V(x, \lambda)$ is a Lyapunov function in $\mathcal{B}_{x}$ for all $\lambda \in \mathcal{B}_{\lambda}$ and $\tau \in \mathcal{B}_{\tau}$. Thus, from Lemma 1 , the set $\mathcal{R}$ will be invariant if in addition the condition $\mathcal{R} \subset \mathcal{B}_{x}$ is satisfied for all $\lambda \in \mathcal{B}_{\lambda}$.

Using the $\mathcal{S}$-Procedure, the condition $\mathcal{R} \subset \mathcal{B}_{x}$ can be checked by the following set of constraints:

$$
2\left(1-a_{k}^{\prime} x\right)+x^{\prime} \mathcal{P}(x, \lambda) x-1 \geq 0, \forall(x, \lambda) \in \mathcal{B}_{x} \times \mathcal{B}_{\lambda}, \forall k
$$

Taking into account the definition of the Lyapunov matrix in (15), the above is equivalent to:

$$
\left.\left[\begin{array}{c}
1 \\
\Theta x \\
x
\end{array}\right]^{\prime}\left[\begin{array}{c}
1 \\
0 \\
a_{k}
\end{array}\right] \quad \begin{array}{cc}
0 & a_{k}^{\prime}
\end{array}\right]\left[\begin{array}{c}
1 \\
\Theta x \\
x
\end{array}\right] \geq 0,
$$

for all $k \in\left\{1, \ldots, n_{e}\right\}$, where $\Theta=\Theta(x, \lambda)$.

Keep in mind that the sector bound condition in (7) is guaranteed if the condition $\mathcal{R} \subset \mathcal{X}_{\rho}$ holds for all $(x, \lambda) \in \mathcal{B}_{x} \times \mathcal{B}_{\lambda}$. By the same arguments, $\mathcal{R}$ belongs to $\mathcal{X}_{\rho}$ if the following is satisfied:

$$
\left[\begin{array}{c}
1 \\
\Theta x \\
x
\end{array}\right]^{\prime}\left[\begin{array}{cc}
(1+\rho)^{2} & {\left[\begin{array}{cc}
0 & K^{\prime}
\end{array}\right]} \\
{\left[\begin{array}{c}
0 \\
K
\end{array}\right]} & P
\end{array}\right]\left[\begin{array}{c}
1 \\
\Theta x \\
x
\end{array}\right] \geq 0
$$

for a given $\rho \geq 0$.

From above analysis, we can infer that (22) and (23) imply the following

$$
\mathcal{R}=\left\{x: V(x, \lambda) \leq 1, \lambda \in \mathcal{B}_{\lambda}\right\} \subset \mathcal{B}_{x} \cap \mathcal{X}_{\rho}
$$

Remark 4 It should be noted that a bad guess for $\rho$ may lead to serious conservativeness on estimating the SR. A possible solution to this problem is to define the shape of $\mathcal{B}_{x}$, perhaps based on physical reasoning as proposed in Section 5, and then choose $\rho$ sufficiently large such that $\mathcal{B}_{x} \subset \mathcal{X}_{\rho}$. Whenever there is no specific information about the size and shape of $\mathcal{B}_{x}$ we can define it as follows

$$
\mathcal{B}_{x}=\left\{x:\left|x_{i}\right| \leq \alpha, i=1, \ldots, n\right\}
$$

and use the parameter $\alpha$ (a scaling factor) to iteratively adjust its size so that $\mathcal{R}$ is maximized.

Remark 5 Notice that the size of $\mathcal{R}$ is related with the pnorm of the matrix $\mathcal{P}(x, \lambda)$. More precisely, as large is $\|\mathcal{P}(x, \lambda)\|_{p}$ smaller will be the values of $x$ can take such that $x^{\prime} \mathcal{P}(x, \lambda) x \leq 1$ is satisfied. Normally, we minimize the trace norm (or simply the trace function for a symmetric matrix) in order to maximize the size of $\mathcal{R}$ Kiyama and Iwasaki (2000). However, the minimization of $\operatorname{trace}(\mathcal{P}(x, \lambda))$ is a nonconvex problem since the Lyapunov matrix is a quadratic function of $x$ and $\lambda$. To overcome this problem, we will approximately maximize the size of $\mathcal{R}$ by means of the following optimization problem:

$$
\min _{P, R} \text { trace } \Pi(P, R): \text { (22), (23), (3) and (8). }
$$

where $\Pi(P, R)=P+R N+N^{\prime} R^{\prime}, R$ is a free multiplier to be determined and $N=N(x, \lambda)$ is an affine matrix function of $(x, \lambda)$ specified in next section such that

$$
N(x, \lambda)\left[\begin{array}{c}
\Theta(x, \lambda) \\
I_{n}
\end{array}\right] x=0 .
$$


From above, we get the following

$$
x^{\prime}\left[\begin{array}{c}
\Theta(x, \lambda) \\
I_{n}
\end{array}\right]^{\prime} \Pi(P, R)\left[\begin{array}{c}
\Theta(x, \lambda) \\
I_{n}
\end{array}\right] x=x^{\prime} \mathcal{P}(x, \lambda) x,
$$

I.e., trace $\Pi(P, R)$ is an approximation of $\operatorname{trace} \mathcal{P}(x, \lambda)$.

\section{STABILITY ANALYSIS}

Before we present the main result of this paper, observe there are some equality constraints associated with the system model representation and the Lyapunov matrix. More specifically, we have:

$\Omega_{1} x+\Omega_{2} \xi=0, \Phi_{1} u+\Phi_{2} \phi=0,\left[\begin{array}{ll}I_{v} & -\Theta\end{array}\right]\left[\begin{array}{c}\Theta \\ I_{n}\end{array}\right] x=0$.

In addition, the use of standard LMI techniques for testing state-dependent matrix inequalities can be quite conservative Trofino (2000). For example, consider the condition:

$$
x^{\prime} \mathcal{T}(x) x>0, \forall x \in \mathcal{B}_{x} .
$$

where $\mathcal{T}(x)$ is asymmetric affine matrix function of $x$. The above condition may be checked by

$$
\mathcal{T}(x)>0, \forall x \in \mathcal{V}\left(\mathcal{B}_{x}\right),
$$

and hence the following is satisfied

$$
y^{\prime} \mathcal{T}(x) y>0, \forall x \in \mathcal{B}_{x}, \forall y \in \mathbb{R}^{n} .
$$

Obviously, this is too conservative. To relax this, the notion of linear annihilators was introduced by Trofino (2000) as below:

Definition 1 A matrix $C(x)$ is called a linear annihilator of $x$ if it is a linear function of $x$ and $C(x) x=0$.

In this paper, we will consider the following linear annihilator:

$$
C(x)=\left[\begin{array}{ccccc}
x_{2} & -x_{1} & 0 & \cdots & 0 \\
0 & x_{3} & -x_{2} & \cdots & 0 \\
\vdots & \vdots & \vdots & \vdots & \vdots \\
0 & \cdots & 0 & x_{n} & -x_{n-1}
\end{array}\right] \in \mathbb{R}^{(n-1) \times n}
$$

The basic idea for incorporating the equality constraints in (25) and $C(x) x=0$ into the stability conditions of Lemma 1 is to associate free multiplier to them by using the well-known Finsler's lemma (Finsler, 1937; Boyd et al., 1994), hence reducing the conservativeness of checking state-dependent LMIs.
For simplicity of notation, consider the following auxiliary matrices:

$$
\begin{aligned}
& E=\left[\begin{array}{ll}
0_{r \times v} & \Omega_{1}
\end{array}\right], F=\left[\begin{array}{cc}
I_{v} & -(\Theta+\tilde{\Theta}) \\
0 & I_{n}
\end{array}\right], \\
& G=\left[\begin{array}{cc}
0 v & 0 \\
0 & A_{1}
\end{array}\right], H=\left[\begin{array}{c}
0 \\
A_{2}
\end{array}\right], J=\left[\begin{array}{c}
0 \\
B_{1}
\end{array}\right], \\
& M=\left[\begin{array}{c}
0 \\
B_{2}
\end{array}\right], N=\left[\begin{array}{cc}
0 & C(x) \\
I_{v} & -\Theta
\end{array}\right] \text {, } \\
& Q=\left[\begin{array}{ccccc}
0 & E & \Omega_{2} & 0 & 0 \\
0 & 0 & 0 & \Phi_{1} & \Phi_{2} \\
0 & N & 0 & 0 & 0 \\
-F & G & H & J & M
\end{array}\right] .
\end{aligned}
$$

where $\Theta=\Theta(x, \lambda)$ and $\tilde{\Theta}=\tilde{\Theta}(x)$.

Then, we can propose the following result for estimating robust stability regions for nonlinear systems with input saturation.

Theorem 2 Consider system (1) with A1-A4 and its representation in (10) with A5. Let $\Theta(x, \lambda)$ be a given affine matrix function of $(x, \lambda)$ and consider the auxiliary matrix $\tilde{\Theta}(x)$ as defined in (19). Let $\mathcal{B}_{x}, \mathcal{B}_{\tau}$ and $\mathcal{B}_{\lambda}$ be given polytopes. Let $\rho \geq 0$ be a given level of over saturation and $K$ a given constant vector such that the closed-loop system in (1) is locally stable. Suppose the matrices $P, R, S, L_{k}$ (for $\left.k=1, \ldots, n_{e}\right), W$, and the positive scalar $\mu$ are a solution to the following optimization problem, where the LMIs are constructed at $\mathcal{V}\left(\mathcal{B}_{x} \times \mathcal{B}_{\tau} \times \mathcal{B}_{\lambda}\right)$.

$$
\begin{aligned}
& \min \operatorname{trace}\left(P+R N+N^{\prime} R^{\prime}\right) \text { subject to: } \\
& P+R N+N^{\prime} R^{\prime}>0, P=P^{\prime} \\
& {\left[\begin{array}{c}
1 \\
\left.\left[\begin{array}{c}
0 \\
a_{k}
\end{array}\right] \quad \begin{array}{cc}
0 & a_{k}^{\prime}
\end{array}\right] \\
\left(P+L_{k} N+N^{\prime} L_{k}^{\prime}\right)
\end{array}\right] \geq 0, \forall k} \\
& {\left[\begin{array}{cccc}
(1+\rho)^{2} & \tilde{K} & \\
\tilde{K}^{\prime} & \left(P+S N+N^{\prime} S^{\prime}\right)
\end{array}\right] \geq 0} \\
& {\left[\begin{array}{ccccc}
0 & P & 0 & 0 & 0 \\
P & -\mu \rho_{2} \tilde{K}^{\prime} \tilde{K} & 0 & \mu \rho_{1} \tilde{K}^{\prime} & 0 \\
0 & 0 & 0 & 0 & 0 \\
0 & \mu \rho_{1} \tilde{K} & 0 & -\mu & 0 \\
0 & 0 & 0 & 0 & 0
\end{array}\right]+}
\end{aligned}
$$

where $\rho_{1}$ and $\rho_{2}$ are given by (9) and $\tilde{K}=\left[\begin{array}{ll}0 & K^{\prime}\end{array}\right]$.

Then, $V(x, \lambda)$ is a Lyapunov function in $\mathcal{B}_{x} \times \mathcal{B}_{\lambda}$. Moreover, $\mathcal{R}$ as defined in (21) is an invariant set for all $\lambda \in \mathcal{B}_{\lambda}$, i.e. for all $x(0) \in \mathcal{R}$ the trajectory $x(t)$ belongs to $\mathcal{R}$ and approaches to origin as $t \rightarrow \infty$.

Proof: Suppose that (30), (31), (32) and (33) are satisfied at all vertices of $\mathcal{B}_{x} \times \mathcal{B}_{\tau} \times \mathcal{B}_{\lambda}$. Thus, by convexity, they are also 
satisfied for all $x \in \mathcal{B}_{x}, \tau \in \mathcal{B}_{\tau}$ and $\lambda \in \mathcal{B}_{\lambda}$. For simplicity of notation define the following vector:

$$
\zeta=\left[\begin{array}{c}
\Theta(x, \lambda) x \\
x
\end{array}\right] \in \mathbb{R}^{(n+v)}
$$

Let $\Gamma_{a} \in \mathbb{R}^{n \times(n+v)}$ be a matrix such that $\Gamma_{a} \zeta=x$, e.g. $\Gamma_{a}=\left[\begin{array}{ll}0_{n \times v} & I_{n}\end{array}\right]$, and define

$$
\Gamma_{b}=\left[\begin{array}{lllll}
0_{n \times v} & 0_{n} & \Gamma_{a} & 0_{n \times m} & 0_{n \times(q+1)}
\end{array}\right] .
$$

For convenience, represent the LMI (30) by $\Sigma_{a}>0$. Since this inequality is strict, for some sufficient small positive scalar $\epsilon_{1}$, one can add the term $-\epsilon_{1} \Gamma_{a}^{\prime} \Gamma_{a}$ to $\Sigma_{a}$ without changing its sign, i.e. the condition $\Sigma_{a}-\epsilon_{1} \Gamma_{a}^{\prime} \Gamma_{a} \geq 0$ is still satisfied. Pre- and post-multiplying $\Sigma_{a}-\epsilon_{1} \Gamma_{a}^{\prime} \Gamma_{a} \geq 0$ by $\zeta^{\prime}$ and $\zeta$, respectively, we get

$$
\epsilon_{1} x^{\prime} x \leq \zeta P \zeta=V(x, \lambda), \forall(x, \lambda) \in \mathcal{B}_{x} \times \mathcal{B}_{\lambda}
$$

since by construction

$$
N \zeta=\left[\begin{array}{cc}
0 & C(x) \\
I_{v} & -\Theta(x, \lambda)
\end{array}\right]\left[\begin{array}{c}
\Theta(x, \lambda) \\
I_{n}
\end{array}\right] x=0
$$

Keep in mind that $(x, \lambda)$ belongs to $\mathcal{B}_{x} \times \mathcal{B}_{\lambda}$, thus the elements of $N$ and $\Sigma_{a}$ are bounded. As a result, there exists a sufficient large positive scalar $\epsilon_{a}$ such that $\epsilon_{a} \zeta^{\prime} \zeta \geq \zeta^{\prime} \Sigma_{a} \zeta$ that in turns yields $\epsilon_{a}\left(x^{\prime} x+x^{\prime} \Theta^{\prime} \Theta x\right) \geq \zeta^{\prime} P \zeta$. Also, there exists a sufficient large positive scalar $\epsilon_{b}$ such that $\epsilon_{b} I_{n} \geq \Theta^{\prime} \Theta$. Hence,

$$
V(x, \lambda)=\zeta^{\prime} P \zeta \leq \epsilon_{2} x^{\prime} x=\epsilon_{a}\left(1+\epsilon_{b}\right) x^{\prime} x,
$$

for all $(x, \lambda) \in \mathcal{B}_{x} \times \mathcal{B}_{\lambda}$.

Now, consider the LMI in (33). For simplicity, we represent it by $\Sigma_{b}<0$. Since this LMI is strict, for some sufficient small positive scalar $\epsilon_{3}$, one can add the term $\epsilon_{3} \Gamma_{b}^{\prime} \Gamma_{b}$ to $\Sigma_{b}$ without changing the sign, i.e. the condition $\Sigma_{b}+\epsilon_{3} \Gamma_{b}^{\prime} \Gamma_{b} \leq 0$ is also satisfied. Pre-multiplying it by $\left[\begin{array}{lllll}\dot{\zeta}^{\prime} & \zeta^{\prime} & \xi^{\prime} & u^{\prime} & \phi^{\prime}\end{array}\right]$ and post-multiplying by its transpose leads to:

$$
\begin{aligned}
& {\left[\begin{array}{c}
\dot{\zeta} \\
\zeta \\
\xi \\
u \\
\phi
\end{array}\right]^{\prime}\left[\begin{array}{ccccc}
0 & \star & 0 & 0 & 0 \\
P & -\mu \rho_{2} \tilde{K}^{\prime} \tilde{K} & 0 & \star & 0 \\
0 & 0 & 0 & 0 & 0 \\
0 & \mu \rho_{1} \tilde{K} & 0 & -\mu & 0 \\
0 & 0 & 0 & 0 & 0
\end{array}\right]\left[\begin{array}{l}
\dot{\zeta} \\
\zeta \\
\xi \\
u \\
\phi
\end{array}\right] \leq-\epsilon_{3} x^{\prime} x} \\
& \forall\left\{\begin{array}{l}
x \in \mathcal{B}_{x} \\
\tau \in \mathcal{B}_{\tau} \\
\lambda \in \mathcal{B}_{\lambda}
\end{array}\right\}:\left\{\begin{array}{l}
-F \dot{\zeta}+G \zeta+H \xi+J u+M \phi=0, \\
N \zeta=0, \\
\Omega_{1} x+\Omega_{2} \xi=0, \\
\Phi_{1} u+\Phi_{2} \phi=0 .
\end{array}\right.
\end{aligned}
$$

From (17) and (18), the time-derivative of $\Theta(x, \lambda) x$ is given by:

$$
\frac{d(\Theta(x, \lambda) x)}{d t}=(\tilde{\Theta}(x)+\Theta(x, \lambda)) \dot{x}
$$

It is easy to verify that the above equality and (10) have the compact form $F \dot{\zeta}=G \zeta+H \xi+J u+M \phi$. Also, from (10) and (36), note that $\Omega_{1} x+\Omega_{2} \xi=0, \Phi_{1} u+\Phi_{2} \phi=0$ and $N \zeta=0$, respectively. These relations can be rewritten as $Q\left[\begin{array}{lllll}\dot{\zeta}^{\prime} & \zeta^{\prime} & \xi^{\prime} & u^{\prime} & \phi^{\prime}\end{array}\right]^{\prime}=0$.

Hence, the inequality (38) is equivalent to the following:

$$
\dot{V}(x, \lambda)-\mu\left(u-K^{\prime} x\right)\left(u-\rho_{2} K^{\prime} x\right) \leq-\epsilon_{3} x^{\prime} x
$$

From (7), $\mu\left(u-K^{\prime} x\right)\left(u-\rho_{2} K^{\prime} x\right) \leq 0$ for all $x \in \mathcal{X}_{\rho}$. As a result, we have that

$$
\dot{V}(x, \lambda) \leq-\epsilon_{3} x^{\prime} x, \forall x \in \mathcal{B}_{x} \cap \mathcal{X}_{\rho}, \tau \in \mathcal{B}_{\tau}, \lambda \in \mathcal{B}_{\lambda}
$$

Then, from (35), (37) and (39) the system is locally exponentially stable.

Now, consider (31) and (32). Pre- and post multiplying (31) by [ $\left.\begin{array}{lll}1 & x^{\prime} \Theta^{\prime} & x^{\prime}\end{array}\right]$ and its transpose, respectively, yields (22). Similarly, (32) implies (23). Then, $\mathcal{R}$ is a positively invariant set, i.e. for all $x(0) \in \mathcal{R}$ the trajectory $x(t) \in \mathcal{R}$ and approaches the origin as $t \rightarrow \infty$.

Remark 6 The method proposed in this paper only considers the single-input and single-output case. However, we can easily extend this technique to deal with multi-loop systems, if the saturation operator has a decoupled structure. In this case, the saturation vector (with $w$ elements) is given by:

$$
\operatorname{sat}\left(K^{\prime} x\right) \triangleq\left[\begin{array}{lll}
\operatorname{sat}\left(K_{1}^{\prime} x\right) & \cdots & \operatorname{sat}\left(K_{w}^{\prime} x\right)
\end{array}\right]
$$

where $K_{i} \in \mathbb{R}^{n}, i=1, \ldots, w$, refers to the $i$-th row of the gain matrix $K \in \mathbb{R}^{w \times n}$. Then, we can apply Theorem 2 taking into account the $w$ input channels by considering $w$ constraints $\mathcal{R} \subset \mathcal{X}_{\rho_{i}} \triangleq\left\{x:\left|K_{i}^{\prime} x\right| \leq\left(1+\rho_{i}\right)\right\}$.

Remark 7 The choice of the matrix $\Theta(x, \lambda)$ defines the complexity of $V(x, \lambda)$ in (15). The more general Lyapunov function is obtained by defining $\Theta(x, \lambda)$ as follows:

$$
\Theta(x, \lambda)=\left[\begin{array}{llllll}
x_{1} I_{n} & \cdots & x_{n} I_{n} & \lambda_{1} I_{n} & \cdots & \lambda_{p} I_{n}
\end{array}\right]^{\prime} .
$$

However, large dimensions of $\Theta(x, \lambda)$ leads to a more intensive computation that can be sometimes prohibitive because of the system dimension.

Remark 8 The conservativeness of estimating stability regions (SRs) in our approach depends on the size and shape of the overbounding polytope $\mathcal{B}_{x}$. A possible solution could be obtained by taking into account the qualitative behavior of the nonlinear systems by means of the bifurcation theory Seydel (1994). Unstable equilibrium points, eigenvalues and eigenvectors give important information about the directions of trajectories close to the boundary of the true domain of attraction and can be used to determine the size and shape of $\mathcal{B}_{x}$. A simple way to use this information will be given in next section. 


\section{NUMERICAL EXAMPLE}

In order to illustrate the proposed approach, we analyze in the following the stability of the origin of system (11) defined in Example 1.

To this end, assume for system (11) that $\mathcal{B}_{\lambda}=[0.9,1.1]$ and $K^{\prime}=\left[\begin{array}{ll}-2 & 0\end{array}\right]$. Also, consider its representation in (14) and define the Lyapunov function candidate by choosing:

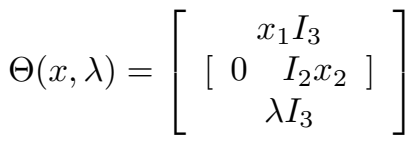

The equilibrium points of system (11) are given:

$$
\bar{x}_{2}=0 \text { and } \lambda \sin \bar{x}_{1}=\operatorname{sat}\left(2 \bar{x}_{1}\right)
$$

where $\left(\bar{x}_{1}, \bar{x}_{2}\right)$ represents the stationary solutions. Notice that the number of equilibrium points will depend on the values of $\lambda \in \mathcal{B}_{\lambda}$ that lead to different possible solutions for $\lambda \sin \bar{x}_{1}-\operatorname{sat}\left(2 \bar{x}_{1}\right)=0$, see a graphical interpretation of this equation in Figure 1.

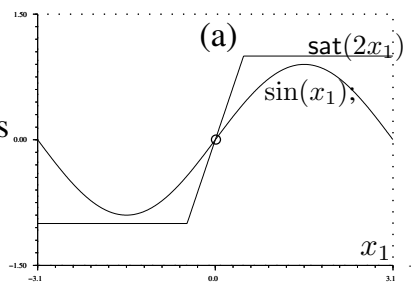

(c)

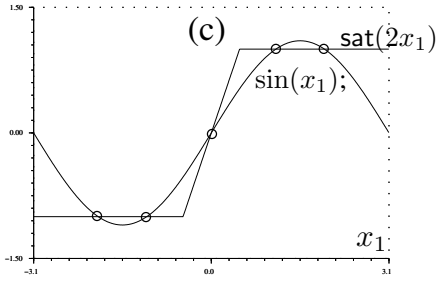

Figure 1: Equilibrium points equation: (a) $\lambda=0.9$, (b) $\lambda=$ 1 and (c) $\lambda=1.1$.

Also, the Jacobian matrix of system (11) is as follows:

$$
\mathbf{A}(\bar{x})=\left[\begin{array}{cc}
0 & 1 \\
\left(\lambda \cos x_{1}-\frac{\partial \operatorname{sat}\left(2 x_{1}\right)}{\partial x_{1}}\right) & -1
\end{array}\right]_{x=\bar{x}}
$$

where $\bar{x}$ refers to the state vector evaluated at the equilibrium point.

Analyzing Figure 1 and taking into account (41), we have the following cases:

(a) $\lambda=0.9 \Rightarrow$ one stable equilibrium point at system origin; (b) $\lambda=1.0 \Rightarrow$ three equilibrium points at $(0,0)$ (stable) and $( \pm \pi / 2,0)$ (non-hyperbolic points, see e.g. Seydel (1994));

(c) $\lambda=1.1 \Rightarrow$ five equilibrium points at $(0,0),( \pm 2.0,0)$ (stables) and $( \pm 1.14,0)$ (unstables).

Clearly, (c) is the worst-case for estimating the stability region in which the domain of attraction of $(0,0)$ is bounded by two unstable equilibrium points at $(1.14,0)$ and $(-1.14,0)$. As these points are symmetrical with respect to the origin, both have the same Jacobian matrix which is given bellow:

$$
\mathbf{A}(( \pm 1.14,0))=\left[\begin{array}{cc}
0 & 1 \\
0.46 & -1
\end{array}\right]
$$

and associated with above matrix, we have the eigenvalues $\sigma_{1}=0.34, \sigma_{2}=-1.34$ (characterizing a saddle point), and the following eigenvectors:

$$
v_{1}=\left[\begin{array}{l}
0.95 \\
0.32
\end{array}\right] \text { and } v_{2}=\left[\begin{array}{r}
-0.60 \\
0.80
\end{array}\right] \text {. }
$$

The above (real) eigenvectors have a geometrical meaning Seydel (1994). In fact, they define two straight lines passing through $( \pm 1.14,0)$ and each half-ray is a trajectory of the following linearized dynamics of $(11)$ at $( \pm 1.14,0)$ :

$$
\dot{z}=\mathbf{A}(( \pm 1.14,0)) z
$$

where $z=x-\bar{x}$. For the nonlinear problem, the eigenvector $v_{2}$ associated with the stable eigenvalue $\sigma_{2}$ defines the tangent to the incoming trajectories (stable manifold or insets) at $( \pm 1.14,0)$ and thus gives the approximate direction of the separatrix.

From the above analysis, we can construct the overbounding set $\mathcal{B}_{x}$ by taking into account the unstable equilibrium points $( \pm 1.14,0)$ and the eigenvector $v_{2}$ in (43) leading to the polytope in Figure 2 (only represented in $x_{1}, x_{2}$ sub-space) which is defined by the following set of vertices:

$$
\begin{gathered}
\left\{\left[\begin{array}{r}
a \\
-b \\
c
\end{array}\right],\left[\begin{array}{l}
a \\
0 \\
c
\end{array}\right],\left[\begin{array}{r}
-a \\
b \\
c
\end{array}\right],\left[\begin{array}{r}
-a \\
0 \\
c
\end{array}\right],\right. \\
\left.\left[\begin{array}{r}
a \\
-b \\
-c
\end{array}\right],\left[\begin{array}{r}
a \\
0 \\
-c
\end{array}\right],\left[\begin{array}{r}
-a \\
b \\
-c
\end{array}\right],\left[\begin{array}{r}
-a \\
0 \\
-c
\end{array}\right]\right\}
\end{gathered}
$$

where $a=1.14, b=2.67$ and $c=\sin (a)$.

In accordance with (44), define the admissible values of $\tau$ in (14) as follows:

$$
\mathcal{B}_{\tau}=\left[0, \sqrt{1-c^{2}}\right]
$$




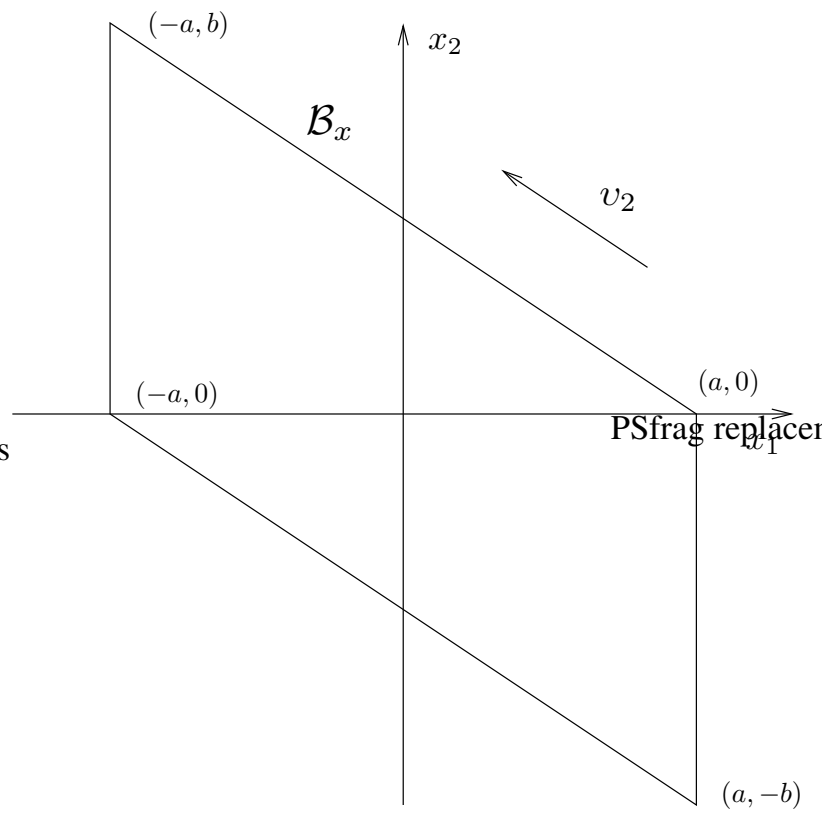

Figure 2: Overbounding polytope $\mathcal{B}_{x}$.

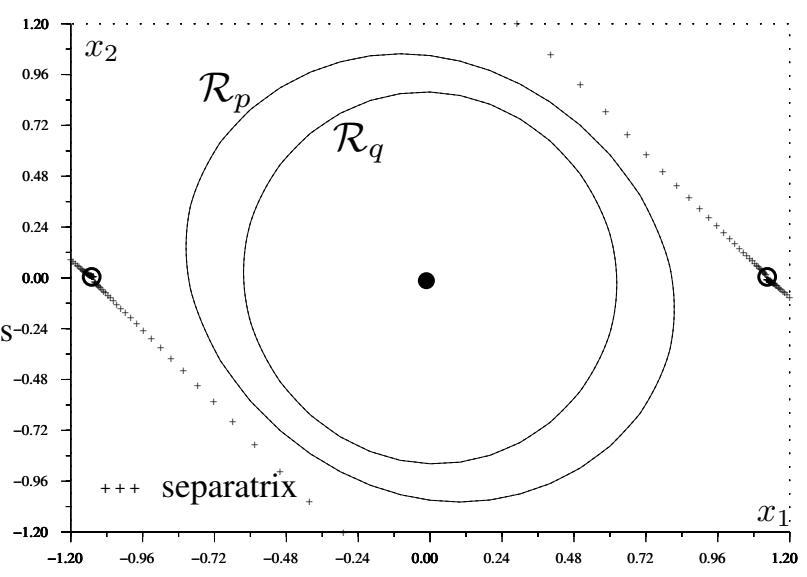

Figure 3: Estimates of SR: $\mathcal{R}_{q}$ and $\mathcal{R}_{p}$.

For comparison purposes, we will consider to determine the stability region of system (11) the following partition for the matrix $P$ in (15):

$$
P=\left[\begin{array}{ll}
P_{2} & P_{1} \\
P_{1}^{\prime} & P_{0}
\end{array}\right], P_{2} \in \mathbb{R}^{v \times v}, P_{0} \in \mathbb{R}^{n \times n}
$$

From above, we can obtain

i. Quadratic Lyapunov function: take $P_{0}$ as a free matrix and set $P_{2}=0, P_{1}=0$.

ii. Polynomial Lyapunov function: consider $P_{0}, P_{1}$ and $P_{2}$ as free matrices.

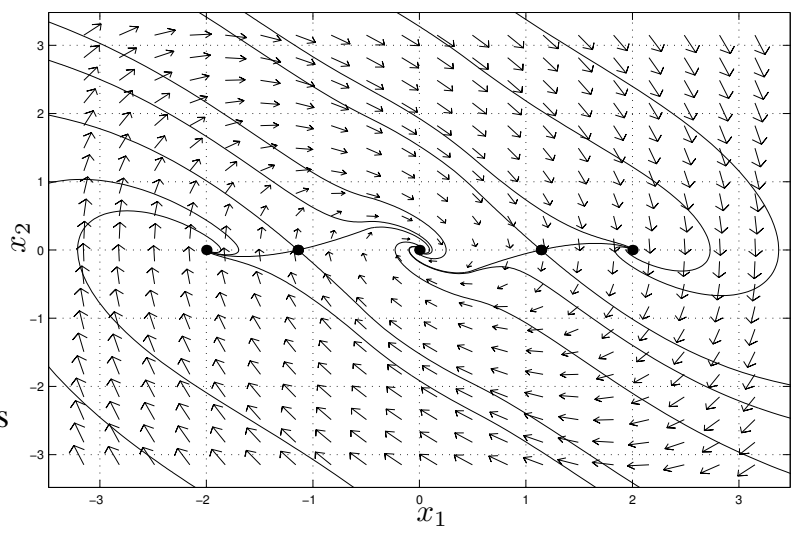

Figure 4: Phase portrait of system (11)

Figure 3 shows estimates of the stability region of system (11) for an optimal $\rho=2.0$ where $\mathcal{R}_{q}$ was obtained with a quadratic Lyapunov function and $\mathcal{R}_{p}$ with a polynomial one. As expected, the polynomial Lyapunov function achieved the best estimate of SR thus justifying the required extra computation.

Also, we give in Figure 4 the phase portrait of system (11) with $\lambda=1.1$ (the worst case for the real domain of attraction). Notice that the SR of the origin is unbounded (and nonconvex) and our method can only estimate closed sets (convex regions) which justifies the conservative result. However, the proposed technique is potentially less conservative than the methods that consider quadratic Lyapunov functions (circle criterion) and can handle uncertainties on the system dynamics.

\section{CONCLUDING REMARKS}

This paper has proposed a convex approach to deal with the problem of estimating robust stability regions for a class of uncertain nonlinear systems subject to input saturation. To this end, the system dynamics is described by means of rational differential-algebraic equations and the saturation nonlinearity is modelled by a sector bound condition similarly to the circle criterion. Also, we have used polynomial Lyapunov functions where the Lyapunov matrix is a quadratic function of state and uncertain parameters in order to obtain less conservative results than the ones that consider quadratic Lyapunov functions. Through a relaxation technique, we give sufficient LMI conditions that assure the local stability of the saturated system and provide an estimate of its stability region that belongs to a polytopic region of the state. The methodology has been applied to an uncertain controlled pendulum with saturation and we have given some remarks about the construction of the overbounding polytope. How- 
ever, the authors are studying a systematic way of defining the differential-algebraic representation of nonlinear system and also the state domain (the region in which the stability conditions are analyzed) in order to turn the proposed approach more appealing to the control community.

\section{REFERENCES}

Barreiro, A., Aracil, J. and Pagano, D. (2002). Detection of Attraction Domains of Nonlinear Systems Using the Bifurcation Analysis and Lyapunov Functions, International Journal of Control 75(5): 314-327.

Bean, S. P., Coutinho, D. F., Trofino, A. and Cury, J. E. R. (2002). Regional Stability of a Class of Nonlinear Hybrid Systems: An LMI Approach, Proceedings of the 41th IEEE Conference on Decision and Control, Las Vegas.

Bender, D. J. and Laub, A. J. (1997). The Linear Quadratic Optimal Regulator for Descriptor Systems, IEEE Transactions on Automatic Control 32(8): 672688.

Boyd, S., Ghaoui, L. E., Feron, E. and Balakrishnan, V. (1994). Linear matrix inequalities in systems and control theory, SIAM books.

Chesi, G., Tesi, A. and Vicino, A. (2002). Computing Optimal Quadratic Lyapunov Functions for Polynomial Nonlinear Systems via LMIs, Proceedings of the 15th IFAC World Congress, Barcelona, Spain.

Coutinho, D. F., Bazanella, A. S., Trofino, A. and Silva, A. S. (2002). Polynomial Lyapunov Functions for a Class of Differential-Algebraic Systems, Proceedings of the XIV Congresso Brasileiro de Automática, Natal, Brazil, pp. $110-115$.

Coutinho, D. and Trofino, A. (2002). Análise de Sistemas Não Lineares Incertos: uma Abordagem LMI, Revista Controle e Automação 13(2): 94-104.

Coutinho, D., Trofino, A. and Fu, M. (2002). Guaranteed Cost Control of Uncertain Nonlinear Systems via Polynomial Lyapunov Functions, IEEE Transactions on Automatic Control 47(9): 1575-1580.

Dussy, S. and El Ghaoui, L. (1997). Multi Objective Bounded Control of Uncertain Nonlinear Systems: An Inverted Pendulum Example, Lectures Notes in Control \& Information Sciences, N. 227. Springer Verlag.

El Ghaoui, L. and Scorletti, G. (1996). Control of rational systems using linear-fractional representations and LMIs, Automatica 32(9): 1273-1284.
Finsler, P. (1937). Über das Vorkommen Definiter and Semidefiniter Formen in Scharen Quadratischer Form, Commentarii Mathematici Helvetici 9: 188-192.

Gomes da Silva Jr., J. M. and Tarbouriech, S. (1999). Invariance and Contractivity of Polyhedra for Linear Countinuous-Time Systems with Saturating Controls, Revista Controle e Automação 10(3): 149-156.

Hindi, H. and Boyd, S. (1998). Analysis of Linear Systems with Saturation using Convex Optimization, Proceedings of the 37th IEEE Conference on Decision and Control, Tampa, pp. 903-908.

Huang, Y. and Jadbabaie, A. (1999). Nonlinear $H_{\infty}$ control: an Enhanced Quasi-LPV Approach, Proceedings of the 14th IFAC World Congress, Beijing, China, pp. 85-90.

Huang, Y. and Lu, W.-M. (1996). Nonlinear Optimal Control: Alternatives to Hamilton-Jacobi Equation, Proceedings of the 35th IEEE Conference on Decision and Control, Kobe, Japan, pp. 3942-3947.

Johansen, T. A. (2000). Computation of Lyapunov Functions for Smooth Nonlinear Systems using Convex Optimization, Automatica 36: 1617-1626.

Johansson, M. (2002). Piecewise Quadratic Estimates of Domain of Attraction for Linear Systems with Saturation, Proceedings of the 15th IFAC World Congress, Barcelona, Spain.

Kiyama, T. and Iwasaki, T. (2000). On the Use of Multiloop Circle Criterion for Saturating Control Synthesis, System \& Control Letters 41: 105-114.

Seydel, R. (1994). Practical Bifurcation and Stability Analysis: from equilibrium to chaos, Springer-Verlag.

Trofino, A. (2000). Robust Stability and Domain of Attraction of Uncertain Nonlinear Systems, Proceedings of the American Control Conference, Chicago.

Yakubovich, V. A. (1971). $\mathcal{S}$-procedure in Nonlinear Control Theory, Vestnik Leninggradskogo Universiteta, Ser. Matematika pp. 62-77. 